\title{
High-Energy Lines in Active Galactic Nuclei
}

\author{
Hagai Netzer
}

School of Physics and Astronomy and the Wise Observatory, The Beverly and Raymond Sackler Faculty of Exact Sciences, Tel-Aviv University, Tel-Aviv 69978, Israel

Abstract. The recent observations of a highly ionized, X-ray absorbing gas in active galactic nuclei (AGN) suggest a new nuclear component, the so-called 'warm absorber'. This gas is likely to be at a temperature of $\sim 1-2 \times 10^{5} \mathrm{~K}$ and is most easily detected in the $0.5-10 \mathrm{keV}$ range, where several oxygen absorption edges are often observed.

This review describes the properties of warm absorbers and the relation to other nuclear components, such as the broad-line emitting gas. The stability of the gas is a key issue and analysis shows that it is likely to be thermally stable, at the above mentioned temperature. When successful models are compared to the data, they can be used to infer the column density, composition and level of ionization of the X-ray absorbing gas. They also show that, on top of the strong continuum absorption, the gas must emit X-ray lines that are at the limit of detection by present day X-ray instruments.

New calculations of X-ray emission lines emitted by ionized X-ray absorbers are shown and discussed. Various line equivalent widths are defined and examples are shown over a large range of column density and ionization parameter. The equivalent width of the strongest $0.5-5 \mathrm{keV}$ lines is only a few tens of $\mathrm{eV}$, but in cases of obscured X-ray source, like in Seyfert 2s, the lines are measured against the scattered and diffuse radiation with much larger equivalent widths. The $\mathrm{X}$-ray absorbing and emitting gas is responsible also for a fraction of the observed flux of some UV emission lines. It is also the cause of the detection of several UV absorption lines. The calculations predict that some of those absorption lines, in particular O VI $\lambda 1035$, are very sensitive warm-absorber tracers. Thus, analysis of the combined X-ray and UV properties is the best way to identify the location and properties of this gas.

Understanding the origin and properties of warm X-ray absorbers is a major challenge of AGN research. Several new ideas are briefly discussed, trying to relate the location, mass, and motion of this gas to what is known about other observed nuclear components.

\section{Introduction: The Ingredients}

Our view of active galactic nuclei $(\mathrm{AGN})$ is constantly changing. In particular, the number of ingredients required to explain their observed spectrum is steadily 
growing. Twenty years ago, there were only three: the central continuum source, the broad-line region (BLR), and the narrow-line region (NLR). Ten years later we became aware of a new component, the cold obscuring material (probably in a shape of a torus), and started to seriously considering the possibility of a multiple central source comprised of an accretion disk and a massive black hole. More recently, X-ray observations have revealed yet another component of highly ionized gas. The gas is situated on the line of sight to the center and is detected via strong absorption features over the $0.1-10 \mathrm{keV}$ range. There is clear evidence for such a component in numerous low-luminosity AGN and detailed studies claim that it is close enough to the center to show short-term variability. This component has been named 'warm absorber', a name that does not accurately describe its nature but is nevertheless familiar enough, at least to X-ray observers, to be used here.

The purpose of this review is to describe the properties of AGN highly ionized absorbers and to attempt to answer the fundamental questions about their location, mass, covering fraction, motion, density and temperature. We investigate the implications of having such a component at various locations around the central source and try to predict the expected (yet undetected) Xray emission lines. The relationship between warm absorbers and other observed components is of great interest. In particular, it is likely to be related to the observed ultraviolet (UV) absorption lines and perhaps also to some extreme-UV emission lines. Any general model must combine all these to a coherent picture and one such attempt is made at the end of this contribution.

\section{Warm Absorbers and the Physics of Highly Ionized Gas}

\subsection{The Basic Properties of Warm Absorbers}

Warm AGN absorbers have been studied in numerous papers (e.g. Halpern 1984; Pan, Stewart, \& Pounds, 1990; Turner \& Pounds 1989; Yaqoob \& Warwick 1991; Nandra \& Pounds 1992; Nandra \& Pounds, 1994; Fabian et al. 1994; Fiore et al. 1993; Mathur et al. 1994; Mathur, Elvis, \& Wilkes 1995; George, Turner, \& Netzer, 1995; Reynolds et al. 1995). Theoretical studies of ionized absorbers include, among others, Halpern (1984), Krolik \& Kallman (1984), Ferland \& Rees (1988), Netzer (1993), Mathur et al. (1994), Netzer, Turner, \& George (1994), Reynolds et al. (1995), George, Turner \& Netzer (1995), Krolik \& Kriss (1995), Turner, Netzer, \& George (1996) and Netzer (1996). Much of the following discussion is based on the last reference.

The strongest observed features in warm absorbers are the $\mathrm{O}$ VII absorption edge at $740 \mathrm{eV}$ and the $\mathrm{O}$ VIII edge at $870 \mathrm{eV}$. There are weaker edge-like features and indication of weak emission components (see I. George's contribution in this volume). The level of ionization of this gas is much higher than observed in the BLR, hence the ionization parameter is larger by a factor of 10-100. This indicates smaller distance, and/or lower gas density. The basic picture is, however, similar to what is usually assumed for the BLR: an ensemble of clouds that are ionized by a point-like radiation source. The clouds can be characterized by their hydrogen density $\left(n_{\mathrm{H}}\right)$, hydrogen column density $\left(N_{\text {col }}\right)$, chemical composition and distance from the center $r$. A complete model must first treat a single cloud, solving all the statistical equilibrium and thermal equation, and then combine 
the results of many such clouds in all possible ways, taking into account a range in physical conditions and the geometrical distribution of the clouds.

An important parameter of the model is the integrated covering fraction of the clouds $C_{\mathrm{f}}$. Since we are dealing with absorbing material, this parameter cannot be directly estimated and one must resort to statistical arguments (e.g., the fractional number of AGN showing warm absorbers) or wait for future instruments capable of detecting weak emission lines (see below). The line-of-sight covering fraction must be close to unity otherwise most X-ray absorption would escape detection. While this is only the line-of-sight covering, it is reasonable to assume that generally $C_{\mathrm{f}}>0.5$.

The flux and shape of the central source spectrum are easy to treat since the physics is controlled by the $0.1-10 \mathrm{keV}$ continuum, which is directly observed in many sources. The unobserved, UV part of the continuum is important for correlating the $\mathrm{X}$-ray properties with the predicted and observed UV emission and absorption lines. This, of course, is unknown and introduces some uncertainties.

A fundamental variable of the calculation is the ionization parameter $U$. Best is to define an $\mathrm{X}$-ray ionization parameter, $U_{\mathbf{x}}$, over the restricted $0.1-$ $10 \mathrm{keV}$ energy range,

$$
U_{\mathrm{x}}=\int_{\nu(0.1 \mathrm{keV})}^{\nu(10 \mathrm{keV})} \frac{L_{\nu} / h_{\nu}}{4 \pi r^{2} n_{\mathrm{H}} c} d \nu
$$

where $L_{\nu}$ is the monochromatic luminosity and $c$ is the speed of light. The level of ionization of most $\mathrm{X}$-ray emitting species is directly proportional to $U_{\mathbf{x}}$ and typical values of interest are in the range of $0.1-1$.

\subsection{Temperature and Stability}

The electron temperature of the highly ionized gas depends on the shape of the ionizing continuum, the gas density, chemical composition, and ionization parameter. A question of great importance is the stability of the gas and the range of conditions suitable for the presence of warm absorbers (see Marshall et al. 1993; Krolik \& Kriss 1995; Hamann et al. 1996; Netzer 1996). New and detailed calculations show that a gas exposed to a typical AGN continuum is thermally stable up to a temperature of about $2 \times 10^{5} \mathrm{~K}$, over a large range in $\mathrm{X}$-ray slope and $\mathrm{X}$-ray/UV continuum flux ratio. The gas temperature is an increasing function of the ionization parameter and it then follows that there is a. maximum value of $U_{\mathrm{x}} \approx 0.3$ below which thermally stable gas can exist. This ionization parameter corresponds to a certain level of ionization and analysis of many warm absorbers indicates a level of ionization which is just below this maximal $U_{\mathbf{x}}$. This may be a clue to the nature of warm absorbers, indicating the range of conditions such gas can survive.

It is hard to tell whether a gas with much larger value of $U_{\mathbf{x}}$ is present since such gas is almost totally transparent to the continuum radiation. In addition, the warm absorbing gas need not be stable (Krolik \& Kriss 1995) and various possible scenarios for gas with $U_{\mathrm{x}}$ much exceeding the maximum allowed value have been considered. The important observational constraint forces us to give special consideration to the restricted range of $U_{\mathrm{x}}$ over which most AGN warm absorbers are observed. 


\subsection{Density and Location}

Given the best estimated range in $U_{\mathrm{x}}$, we can estimate the density of the warm absorbing gas, $N_{\mathrm{WA}}$, as a function of its location. The following assumes a 'typical BLR radius' of

$$
R_{B L R} \approx 0.01 L_{44}^{1 / 2} \mathrm{pc}
$$

where $L_{44}$ is the ionizing luminosity in units of $10^{44} \mathrm{erg} \mathrm{s}^{-1}$, (e.g., S. Kaspi's contribution in this volume). The estimated warm absorber $U_{\mathrm{x}}$ can then be normalized to the ionization parameter required to explain the BLR emission lines. This gives

$$
N_{\mathrm{WA}} \approx 3 \times 10^{4} L_{44} R_{\mathrm{pc}}^{-2} \mathrm{~cm}^{-3},
$$

where $R_{\mathrm{pc}}$ is the distance from the center in pc. For example, for a $L_{44}=1$ AGN, the warm absorber density is about $3 \times 10^{10} \mathrm{~cm}^{-3}$ if located $0.001 \mathrm{pc}$ from the center (i.e., inside the BLR) and $3 \times 10^{7} \mathrm{~cm}^{-3}$ if at $0.03 \mathrm{pc}$ (outside the BLR) from the center. These numbers can, eventually, be verified by direct observations.

\subsection{The Spectrum of Warm X-ray Absorbers}

As explained above, present $\mathrm{X}$-ray observations, mostly by $A S C A$, clearly detect the absorption by highly ionized gas in the $0.5-10 \mathrm{keV}$ spectrum of numerous AGN. The range of ionization parameter, as obtained from detailed fitting to the spectrum of about 20 Seyfert galaxies (see I. George's contribution) is not large, $0.05 \leq U_{\mathrm{x}} \leq 0.5$, and the range of column density approximately $10^{21.5}$ to $10^{23} \mathrm{~cm}^{-2}$. Such gas must emit line and continuum radiation and scatter a fraction of the continuum source photons, mainly via thermal electrons. There are, therefore, three major components to consider: absorption, emission, and reflection.

Figure 1 shows the result of a specific warm-absorber model designed to mimic the spectrum of a typical highly ionized gas. The spectrum is dominated by absorption, in particular by $\mathrm{O}^{+6}$ and $\mathrm{O}^{+7}$ absorption edges, but many emission lines are clearly seen. Some bound-free emission edges are strong too, typical of a situation where the electron temperature is low compared with the equilibrium plasma temperature. The relative strength of all emission features depend on the gas column density and the covering fraction since both determine the amount of emitting material. The example shown in Fig. 1 is for the case of $C_{\mathrm{f}}=1$ and is therefore an illustration of the maximum emission expected for the given column.

The reflection component is hard to detect when the central source is directly seen. In our example, the column density is $10^{22.5} \mathrm{~cm}^{-2}$ resulting in a small Compton depth of 0.02 . Plotting the reflected continuum by itself, as shown in the top panel, reveals very strong (by comparison) lines on top of a weak continuum. The X-ray spectrum of some AGN is is completely dominated by reflection. In particular, Seyfert 2 nuclei, with obscured central sources and high ionization reflectors (e.g., NGC 1068) show this phenomenon.

In general, the observed X-ray spectrum represents a combination of lineof-sight absorption with emission and reflection from components whose exact location is not well known. 


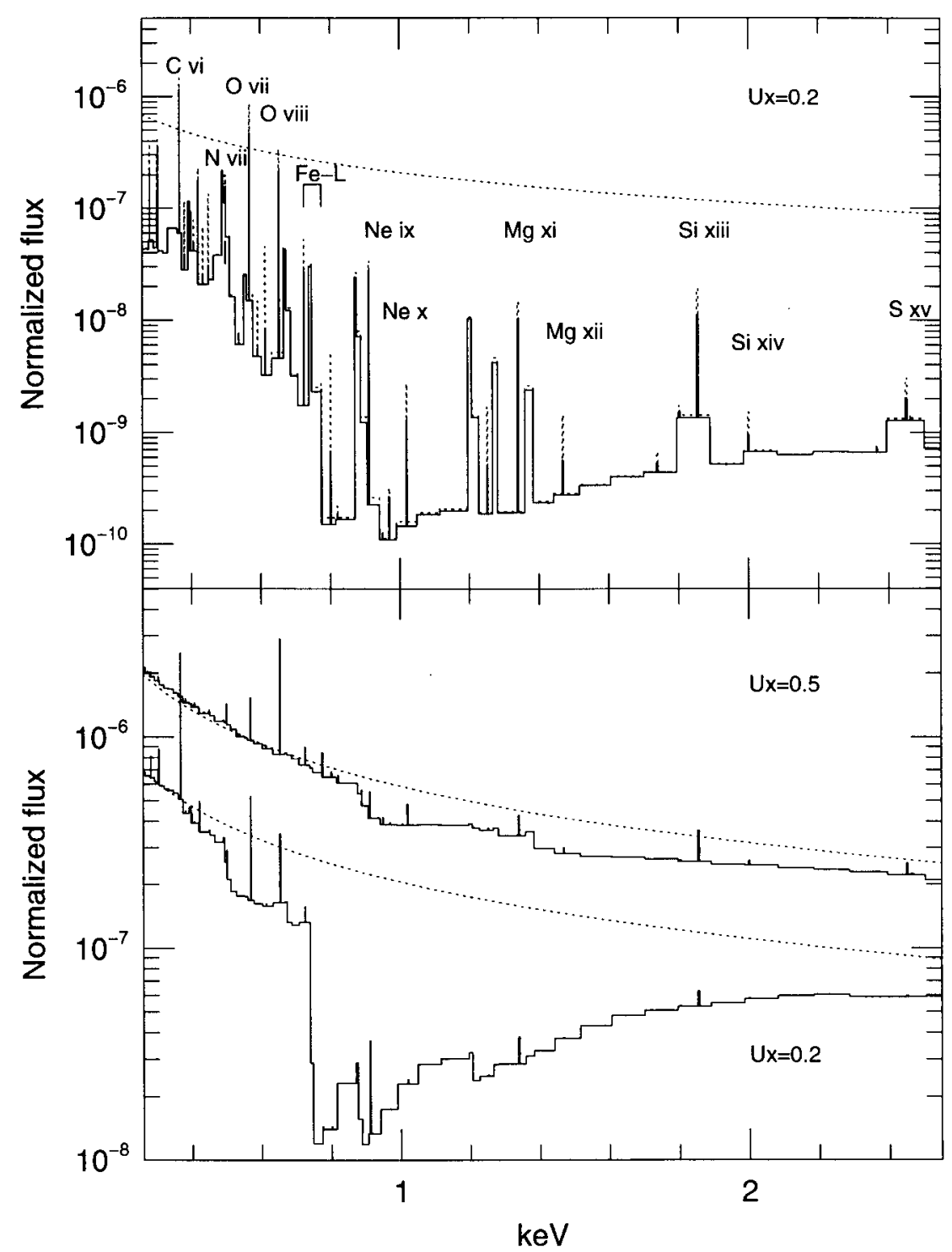

Figure 1. Bottom: Calculated warm-absorber spectra for two values of $U_{\mathbf{x}}$. The dashed line is the incident continuum and the height of the emission lines is fixed by assuming an observed line width of $4000 \mathrm{~km} \mathrm{~s}^{-1}$. Top: the $U_{\mathrm{x}}=0.2$ spectrum as seen for a case where the central continuum is obscured. The dashed line is the same model with an assumed micro-turbulence velocity of $300 \mathrm{~km} \mathrm{~s}^{-1}$. Note the different vertical scale of the bottom and top panels. 


\section{High-Energy Emission Lines}

\subsection{X-Ray Lines and Edges}

Given the conditions (column density, composition, and level of ionization) of the X-ray absorbing gas, one can calculate the expected emission-line spectrum, as shown for example in Fig. 1. The calculations show that H-like and He-like lines of metals with $Z>7$ dominate the $0.5-10 \mathrm{keV}$ spectrum. Examples are O VII $568 \mathrm{eV}, \mathrm{O}$ VIII $653 \mathrm{eV}, \mathrm{Mg}$ XI $1.34 \mathrm{keV}$ and Si XIII $1.85 \mathrm{keV}$. Other lines of significant intensity are the iron Fe-L lines (see the recent paper by Kallman et al. 1996) and the iron $\mathrm{K} \alpha$ lines at $E \geq 6.4 \mathrm{keV}$.

Accurate calculations of line intensities require full solution of the statistical equilibrium equations, allowing for optical depth effects that are likely to be important in warm X-ray, large column-density absorbers. Most strong predicted lines are due to recombination of highly ionized species and collisional excitation is almost negligible due to the low temperature. Fluorescence lines, except for the iron $\mathrm{K} \alpha$ lines, are relatively weak because of the small fluorescence yield. Absorption of continuum photon by resonance lines (the so-called 'continuum fluorescence') must also be considered but the process is of little importance, in particular in those cases where the continuum is not obscured. In some cases, where large velocity gradients are assumed and the central continuum is obscured, this process may contribute to the observed line intensity. Finally, geometry plays a very important role since line photons are subjected to absorption on their way out, especially for large covering fraction geometries.

\subsection{Equivalent Width}

It is customary to describe line intensity using equivalent width $(E W)$ units. Three different types of $E W$ s can be defined:

1. Relative to the incident continuum, $E W($ con $)$. This $E W$ is measured relative to the unattenuated central continuum. It is rarely observed but is a convenient way to compare different lines.

2. Relative to the observed continuum, $E W$ (obs). This is the measured equivalent width which depends on the continuum luminosity and attenuation, the local line and continuum flux, and the reflection of the central continuum radiation.

3. Relative to the gas continuum, $E W$ (gas). This $E W$ is observed when the central X-ray component is obscured, e.g., in Seyfert 2 galaxies. The emission line is measured against the local (free-free, bound-free, and scattered central source) continuum.

Usually $E W$ (gas) is larger than $E W($ con $)$ and $E W($ obs $)$.

$E W($ con) can be estimated from general considerations by counting the number of absorbed ionizing photons and comparing to the continuum flux at the emission-line energy. For example, the maximum $E W($ con $)$ of the $\mathrm{O}$ vill $653 \mathrm{eV}$ line, for an $F_{E} \propto E^{-\alpha_{x}}$ continuum with $\alpha_{x}=0.9$, is about $500 \mathrm{eV}$. Such an $E W$ is an order of magnitude stronger than the upper limit we obtain with $A S C A$. There are several reason for this weak line; absorption by other ions that 
compete with $\mathrm{O}^{+7}$ on the absorption of continuum photons, and absorption of the OVIII $653 \mathrm{eV}$ line photons after they have been emitted and more. The O VIII $653 \mathrm{eV}$ line is always among the strongest predicted emission lines, for the range of conditions thought to be typical of warm absorbers. Thus, the predicted line intensities do not amount to more than a few tens of $\mathrm{eV}$. This calls for the next generation ( $A X A F, X M M, A S T R O-E) \mathrm{X}$-ray instrumentation.

Figure 2 shows the calculated OVIII $653 \mathrm{eV} E W$ (obs), for a grid of models covering a large range in column density and ionization parameter. The covering fraction of the model is unity (full coverage) the density is $10^{10} \mathrm{~cm}^{-3}$ and the composition close to solar. The largest $E W(\mathrm{obs})$ is of the order of $50 \mathrm{eV}$. $E W($ con) in this case (not shown) is very similar to $E W$ (obs). There are, however, some cases where $E W(\mathrm{obs})$ is considerably larger than $E W($ con ), especially when the lines are seen against an attenuated continuum (Netzer 1996).

Figure 3 shows $E W$ (gas) for the same grid of models. In this case, the line is observed against the scattered and diffuse radiation and very large values of $E W$, of order $1 \mathrm{keV}$, are attainable. $E W$ (gas) depends only little on the geometry and $C_{\mathrm{f}}$ since the line flux and the continuum flux against which it is measured, are affected in a similar way by continuum opacity.

\subsection{UV and EUV Emission Lines}

Highly ionized X-ray absorbing gas contributes also to the observed UV line emission. Only two lines, O VI $\lambda 1035$ and Ne VIII $\lambda 774$, are likely to be influenced by that. Detailed calculations show that the maximum contribution to the observed $E W$, given the range of $U_{\mathrm{x}}$ in warm absorbers, is about $5 \AA$ for both and is rather sensitive to the assumed shape of the extreme UV (EUV) continuum. Since typical observed equivalent widths are $5-50 \AA$ for O VI $\lambda 1035$ and $3-10 \AA$ for Ne VIII $\lambda 774$, the latter is likely to be more related to warm X-ray emission. We note, however, that OVI $\lambda 1035$ is stronger in low-luminosity AGN, where warm absorbers seem to be more common.

The idea of a large contribution to O VI $\lambda 1035$ from an optically thin component is not new (Netzer 1976; Shields, Ferland, \& Peterson 1995). These authors assumed the presence of optically thin gas with level of ionization similar to that of the BLR gas. The new warm absorber calculations indicate a different component with much higher ionization. The real situation is likely to be more complex, with a possible coexistence of various components (see below).

Ne VIII $\lambda 774$, is of particular interest since it has recently been observed in several HST-FOS snapshot observations (Hamann, Zou, \& Tytler 1995) and in the quasar PG $1148+549$ (Hamann et al. 1996) with a typical rest equivalent width of $5-10 \AA$. A warm absorber with properties similar to those discussed here can explain a large fraction of its observed intensity provided the covering fraction is close to unity (see Hamann et al. 1996 and Netzer 1996 for more discussion).

Regarding the extreme ultraviolet range, the only published results are by Kaastra, Roos, \& Mewe (1995) who have observed NGC 5548 with the EUVE satellite. These authors obtained a reasonable signal-to-noise spectrum of the $70-100 \AA$ range and claimed to have detected several emission lines. The proposed line identifications (Ne VII $\lambda 88$, Ne VIII $\lambda 88$, and Si VII $\lambda 70$ ) are problematic since no photoionization calculation predicts these lines to be strong. It remains 


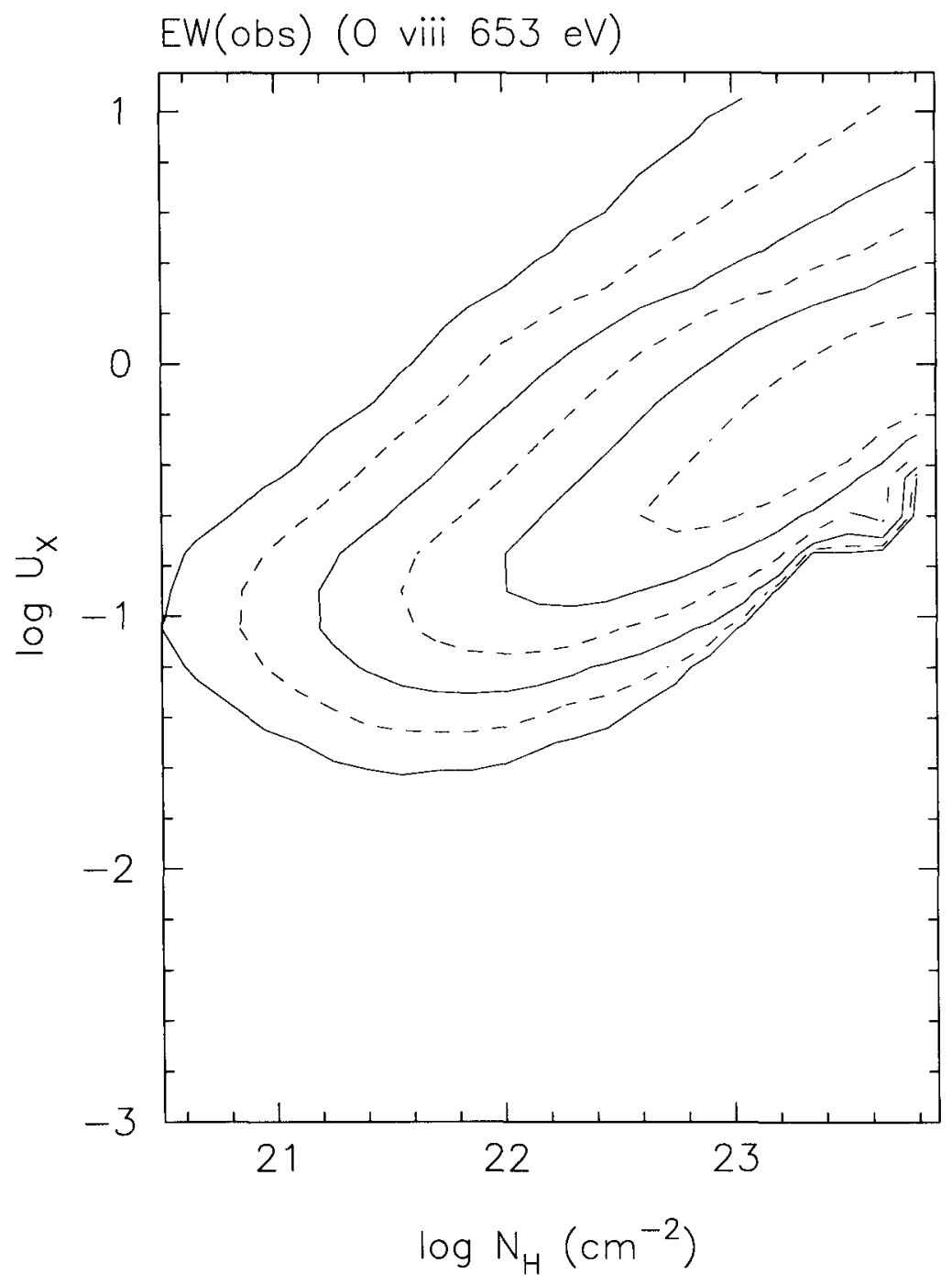

Figure 2. $\quad E W$ (obs) (observed equivalent width) contours for a warm absorber model with an X-ray continuum slope of $\alpha_{x}=0.9$ and gas density of $10^{10} \mathrm{~cm}^{-3}$. Horizontal axis is column density and vertical axis the $0.1-10 \mathrm{keV}$ ionization parameter. Contours at $1,2,4, \ldots \mathrm{eV}$ and largest $E W$ is on the top right. 


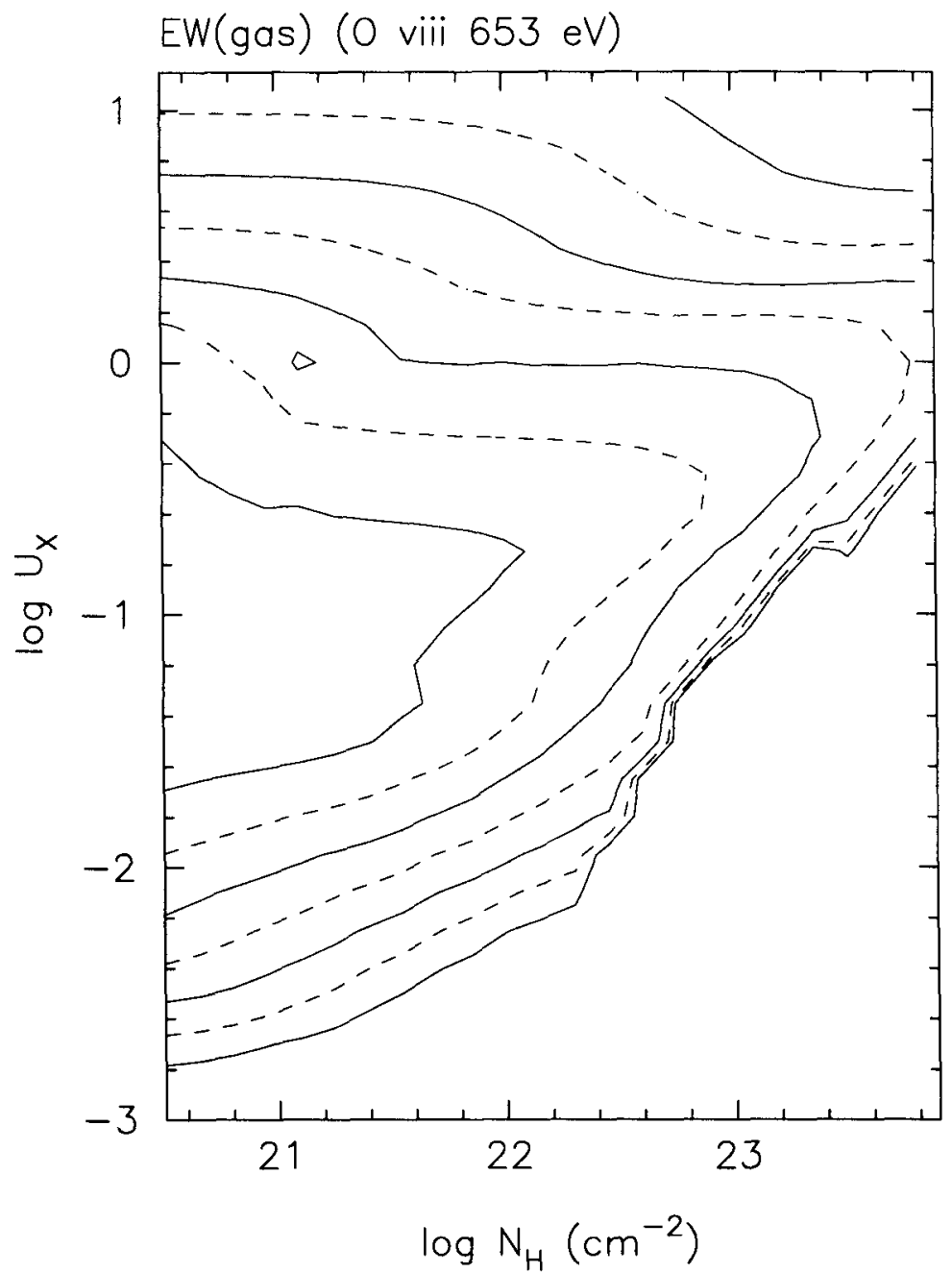

Figure 3. As Figure 2, but for $E W$ (gas) (obscured central source). Contours at $16,32,64 \ldots \mathrm{eV}$ and largest $E W$ is on the left. 
to be seen whether any $70-100 \AA$ emission is related to the presence of warm X-ray absorbers.

\section{Ultraviolet Absorption Lines}

Intrinsic absorption systems are often observed in the $I U E$ spectra of Seyfert 1 galaxies and low-redshift quasars (Ulrich 1988). The IUE statistics are very incomplete and recent $H S T$ observations suggest that the majority of low-luminosity AGN show such features. The absorption components most relevant to our discussion are often blueshifted with respect to the emission lines, by several hundred $\mathrm{km} \mathrm{s}^{-1}$, and they have typical widths (FWHM) of $100-200 \mathrm{~km} \mathrm{~s}^{-1}$, and $E W$ s of the order of $1 \AA$.

Several recent papers (Mathur et al. 1994, Mathur 1994, Mathur, Elvis, \& Wilkes 1995, Kriss et al. 1996) suggested a possible association of such systems with warm X-ray absorbers. $E W$ calculations seem to support this idea at least in a few cases. In all these, the measured C IV $\lambda 1549, \mathrm{~N} \vee \lambda 1240$, and $\mathrm{O}$ VI $\lambda 1035$ absorption lines must represent a trace ionization in a gas component dominated by almost totally stripped carbon, nitrogen, and oxygen. There are, however, well-studied cases (e.g., Kriss et al. 1996) where absorption components indicative of lower ionization, and hence not related to the assumed warm absorber, are detected.

The expected UV line optical depths and $E W$ s can be calculated for the same range of parameters as in Fig. 2. Figure 4 shows one such example pertaining to the $\mathrm{O}$ VI $\lambda 1035$ absorption lines. The diagram shows contour plots of calculated absorption $E W$ for a specific Doppler width of $100 \mathrm{~km} \mathrm{~s}^{-1}$. A comparison with Fig. 2 clearly suggests a region of overlap where both strong X-ray absorption, by $\mathrm{O}$ VII and $\mathrm{O}$ VIII edges $\left(0.05<U_{\mathrm{x}}<0.5\right.$ and a column density exceeding $\left.10^{22} \mathrm{~cm}^{-2}\right)$ and detectable O vi $\lambda 1035$ absorption $(E W(\mathrm{O}$ VI $\lambda 1035) \approx 1 \AA) \mathrm{co-}$ exist. Similar calculations show that $E W(\mathrm{C}$ IV $\lambda 1549)$ is more difficult to detect, and $E W(\mathrm{~N} \vee \lambda 1240)$ is between the above two lines. O VI $\lambda 1035$ is by far the best tracer of warm X-ray absorbers with Ne VIII $\lambda 774$ almost as good. Unfortunately, neither line can be detected by $H S T$ in very low-redshift AGN.

UV absorption lines provide a useful constraint on the location of warm $\mathrm{X}$-ray absorbers. In several well-studied cases, the UV observations suggest a location which is outside the BLR (the absorption line, measured against the blue wing of the emission line, is deeper than the continuum). This may well be the most important clue to the properties of this gas, provided the observed UV lines originate in the same component producing the strong $\mathrm{X}$-ray edges. Simultaneous UV/X-ray studies are thus very promising and likely to contribute to the understanding of this problem, especially if line and edge variability is detected.

\section{Composite Models}

A fundamental issue addressed in this review is the location of the ionized absorbing gas in relation to the other nuclear components. Given the known properties of the BLR, its size (eq. 2) and typical density, we have already obtained 


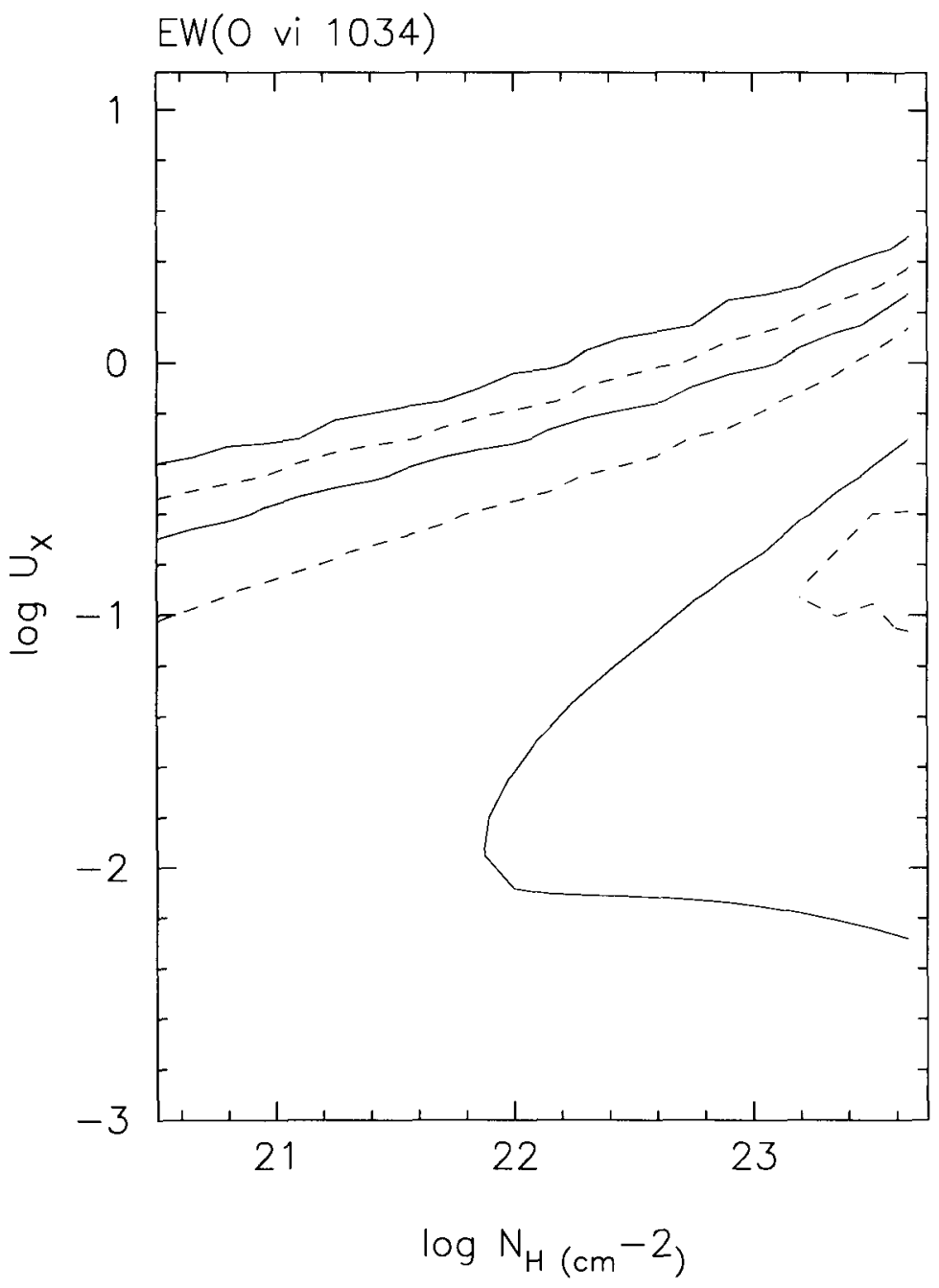

Figure 4. Calculated absorption equivalent widths for Ovi $\lambda 1035$ over the same range of parameters as in Fig. 2. EW contours are at $0.03,0.1,0.3,1.0,3$ and $10 \AA$ and the assumed Doppler parameter $100 \mathrm{~km} \mathrm{~s}^{-1}$. 
an estimate for the typical warm absorber density, $N_{\mathrm{WA}}$ (eq. 3 ). We can use this result to obtain an estimate of the physical thickness of the warm absorber layer, $\Delta R_{\mathrm{WA}}$,

$$
\Delta R_{\mathrm{WA}} \approx 0.1 L_{44}^{-1} N_{22} R_{\mathrm{pc}}^{2} \mathrm{pc},
$$

where $N_{22}$ is the warm-absorber column density in units of $10^{22} \mathrm{~cm}^{-2}$. Many warm absorbers are detected in $L_{44} \approx 1$ AGN and most of them show $N_{22} \geq 1$. This gives, at a typical BLR radius, a thickness of about $10^{13.5} N_{22} \mathrm{~cm}$, at least as large, and probably much larger, than a typical thickness of a BLR cloud. The physical dimension of the warm absorber scales with $R_{\mathrm{pc}}^{2}$ and can be extremely large if situated well outside the BLR. In particular, it fills out all space if $R_{\mathrm{pc}} \approx 10$.

The mass of the warm-absorber component is proportional to its thickness and covering factor and is given by

$$
M_{\mathrm{WA}} \approx 10^{3} N_{22} C_{\mathrm{f}} R_{\mathrm{pc}}^{2} M_{\odot} .
$$

It is comparable to the BLR total mass if the two coexist in the same volume and far exceeds it if located outside of the BLR.

Finally, the recombination time of the warm absorbing gas is given by

$$
t_{\mathrm{rec}} \approx 5 \times 10^{6} T_{5}^{0.7} L_{44}^{-1} R_{\mathrm{pc}}^{2} \mathrm{sec},
$$

where $T_{5}$ is its temperature in units of $10^{5} \mathrm{~K}$. At the $\mathrm{BLR}$ typical radius, it is of the order of $500 \mathrm{sec}$ and at one parsec about one month; i.e., comparable to or longer than the UV continuum variability time scale.

Given the above considerations, we are facing two different possibilities: either the warm gas is inside the BLR or it is well outside of it. The two are very different in terms of the required mass, physical dimension, and recombination time. They raise different questions about the origin of the $\mathrm{X}$-ray absorbing gas, its pressure and temperature and dynamical stage. The following is a simplified suggestion to link all components that is, perhaps, a first step in a long way towards a complete model.

Assume a clumpy BLR with a covering factor of order 0.1 and approximately spherical distribution. The 'clouds' are either bloated stars (BS) with extended winds (Alexander \& Netzer 1994, 1996; see T. Alexander's contribution in this volume) or some high-density condensations losing mass to the intercloud medium. Close to the center of the BS, or condensation, where the density is of the order of $10^{10} \mathrm{~cm}^{-3}$, the emission-line spectrum resembles the one observed in the broad emission lines. Deeper in, there is little contribution to line emission because of the very efficient thermalization and much reduced covering fraction. On the outside, constant evaporation leads to lower-density, higher-ionization gas. At a certain distance from the center of the condensation, this material is totally transparent to the Lyman continuum radiation and the only detectable absorption features are due to highly ionized species, such as $\mathrm{O}^{+7}$.

The highly ionized gas streaming from the condensations fills much of the space between the them and its covering factor approaches unity. The column density remains similar to the column density of the $n_{\mathrm{H}}=10^{10} \mathrm{~cm}^{-3}$ material. Radiation pressure due to the strong radiation of the central source may, at some stage, overcome gravity, starting to push material away from the BLR. 
The decreasing density and increasing level of ionization, may, eventually cause this gas to disappear from sight.

There are no real calculations attempting to model this scenario and it remains to be seen whether the evaporating condensations reach the warm absorber column density and level of ionization as they approach the full covering stage. It is also not clear what is the magnitude of the outward velocity and whether it agrees with the measured velocity of the UV absorption lines. There is also the issue of mass supply over time and other complications. One should note, however, that the gas pressure in warm absorbers, as deduced from the new calculations, is similar to the BLR gas pressure if the two components coexist spatially. This may be a clue to the validity of this toy model.

\section{Open Questions}

The discovery of warm AGN absorbers helps to limit the large number of unknowns related to the physics of such objects. It also raises new questions and issues that must be addressed by future observations and calculations. The following is a list of some questions that are, in my opinion, the most critical.

1. How common are warm absorbers? Is their presence a decreasing function of luminosity?

2. How common are UV absorption lines? Are they detected in all cases where a large column density of X-ray gas is present? If not, why?

3. What are the location and density of warm absorbers? Can we find them by reverberation mapping of X-ray lines and edges?

4. How strong are the iron L-shell lines?

5. How stable is the warm absorbing gas? How important is expansion cooling?

6. What is the covering fraction of the warm absorbing gas? Is it a function of distance from the center?

7. How viable is the 'evaporating condensation' model presented here?

8. Are there several distinct warm-absorber components? This would require the calculations of different geometries where different lines of sight combine emission and absorption in different proportions.

Acknowledgments. Discussions with Ian George, Jane Turner, Tim Kallman, Richard Mushotzky, Paul Nandra, Gary Ferland, Amiel Sterberg, Ari Laor, and Tal Alexander are gratefully acknowledged. This research is supported by USRA at NASA/GSFC, by the Jack Adler Chair of Extragalactic Astronomy and by a special grant of the Israel Science Foundation. 


\section{References}

Alexander, T., \& Netzer, H. 1994, MNRAS, $270,781$.

Alexander, T., \& Netzer, H. 1996, MNRAS, submitted.

Fabian, A. C., Kunieda, H., Inoue, S., Matsuoka, M., Mihara, T., Miyamoto, S., Otani, C., Ricker, G., Tanaka, Y., Yamauchi, M., \& Yaqoob, T. 1994, PASJ, 46, L59.

Ferland, G.J., \& Rees, M. J. 1988, ApJ, 332, 141.

Fiore, F. 1993, ApJ, 415, 129.

George, I. M., Turner, T. J., \& Netzer, H. 1995, ApJ, 438, L67.

Halpern, J. P. 1984, ApJ, 281, 90.

Hamann, F., Zou, L., \& Tytler, D. 1995, ApJ, 444, L69.

Hamann, F., Shields, J. C., Ferland, G. J., \& Korista, K. T. 1996, ApJ, in press.

Kaastra, J.S., Roos, N., \& Mewe, R. 1995, A\&A, 300, 25.

Kallman, T., Liedahl, D., Osterheld, A., Golstein, W., \& Kahn, S. 1996, ApJ, in press.

Kriss, G. A., Espey, B. R., Krolik, J. H., Tsvetanov, Z., Zheng, W., \& Davidsen, A. F. 1996, ApJ, in press.

Krolik, J., \& Kallman, T. 1984, ApJ, 286, 355.

Krolik, J., \& Kriss, G. 1995, ApJ, 447, 512.

Mathur, S. 1994, ApJ, 431, L75.

Mathur, S., Wilkes, B., Elvis, M., Fiore, F. 1994, ApJ, 434, 493.

Mathur, S., Elvis, M., \& Wilkes, B. 1995, ApJ, 452, 230.

Marshall, F.E., et al. 1993, ApJ, 405, 168.

Nandra, K., \& Pounds, K. 1992, Nature, 359, 215.

Nandra, K., \& Pounds, K. 1994, MNRAS, 268, 405.

Netzer, H. 1976, MNRAS, 177, 473.

Netzer, H. 1993, ApJ, 411, 594.

Netzer, H. 1996, ApJ, in press.

Netzer, H., Turner, T. J., \& George, I. M. 1994 ApJ, 435, 106.

Pan, H., Stewart, G. C., \& Pounds, K. A. 1990, MNRAS, 242, 177.

Reynolds, C.S., \& Fabian, A.C. 1995, MNRAS, 273, 1167.

Reynolds, C.S., Fabian, A. C., Nandra, K., Inoue, H., Kunieda, H., \& Iwasawa, K. 1996, MNRAS, in press.

Reynolds, C.S., Fabian, A.C., \& Inoue, H., 1996, MNRAS, in press.

Shields, J. C., Ferland, G. J. \& Peterson, B. M. 1995, ApJ, 441, 507.

Turner, T.J., Nandra, K., George, I. M., Fabian, A.C., \& Pounds, K. A. 1993, ApJ, 419, 127.

Ulrich, M.-H. 1988, MNRAS, 230, 121.

Yaqoob, T., \& Warwick, R.S. 1991, MNRAS, 248, 773. 